\section{Kidney Blood Pressure Research}

\title{
Dietary Salt Intake is a Significant Determinant of Impaired Kidney Function in the General Population
}

\author{
Tomonori Sugiura $^{a} \quad$ Hiroyuki Takase $^{\mathrm{b}} \quad$ Nobuyuki Ohte Yasuaki Dohic $^{\mathrm{a}}$ \\ aDepartment of Cardio-Renal Medicine and Hypertension, Nagoya City University Graduate School \\ of Medical Sciences, Nagoya, 'Department of Internal Medicine, Enshu Hospital, Hamamatsu, \\ 'Department of Internal Medicine, Faculty of Rehabilitation, Nagoya Gakuin University, Seto, Japan
}

\section{Key Words}

Salt intake $\bullet$ Impaired kidney function $\bullet$ Chronic kidney disease $\bullet$ Estimated glomerular filtration rate

\begin{abstract}
Background/Aims: Kidney dysfunction is an important risk factor for cardiovascular disease and end-stage renal disease. This study investigated whether dietary salt intake predicts deterioration of kidney function in the general population. Methods: In all, 12126 subjects with a normal estimated glomerular filtration rate (eGFR $\geq 60 \mathrm{~mL} / \mathrm{min}$ per $1.73 \mathrm{~m}^{2}$ ) attending an annual check-up were enrolled in the study and were followed-up for a median of 1754 days; the endpoint was the development of impaired kidney function (eGFR $<60 \mathrm{~mL} / \mathrm{min}$ per $1.73 \mathrm{~m}^{2}$ ). Individual salt intake was estimated using spot urine analysis. Results: At baseline, mean ( \pm SD) salt intake and eGFR were $10.6 \pm 3.4 \mathrm{~g} /$ day and $80.8 \pm 12.9 \mathrm{~mL} / \mathrm{min}$ per $1.73 \mathrm{~m}^{2}$, respectively. During the follow-up period, 1384 subjects (25.2 per 1000 person-years) developed impaired kidney function. Multivariate Cox hazard regression analysis revealed salt intake as a significant predictor of the new onset of kidney impairment (hazard ratio 1.045; 95\% confidence interval 1.025-1.065). Subjects were divided into two groups based on salt intake; the incidence of impaired kidney function was higher in the group with high than low salt intake $(P<0.001$, log-rank test). Multivariate Cox hazard regression analysis indicated a $29 \%$ increased risk of developing impaired kidney function in the high-salt group. Multivariate linear regression analysis showed a significant correlation between salt intake and yearly decline in eGFR ( $\beta$ $=0.060, P<0.001)$. Conclusion: Salt intake is associated with the development of impaired kidney function in the general population, independent of its effects on blood pressure. Salt restriction may help prevent the development of impaired kidney function.
\end{abstract}




\section{Kidney Blood Pressure Research}

Sugiura et al:: Salt and Chronic Kidney Disease

\section{Introduction}

The number of people with end-stage renal disease (ESRD) is gradually increasing worldwide [1, 2], resulting in an increase in the number of dialysis patients [3], which is a serious economic concern. ESRD is the last stage of chronic kidney disease (CKD). Recently, a new concept of cardiorenal syndrome was proposed based on reports that CKD is one of the important risk factors for cardiovascular disease [4-8], a leading cause of mortality in developed countries. Among patients with CKD, the incidence of cardiovascular death is much more frequent than that of new chronic dialysis [9], indicating the clinical importance of preventing cardiovascular disease among patients with CKD. Furthermore, it is generally difficult to improve impaired kidney function. Thus, primary prevention of CKD must be an important population strategy to prevent cardiovascular diseases.

Several factors, such as hypertension, impaired glucose tolerance, dyslipidemia, obesity, aging, and smoking, have been reported as risk factors for CKD [10-13]. Aging is one factor that has a considerable effect on the deterioration of kidney function in healthy individuals [14]. Although aging is unavoidable, other risk factors can be ameliorated, at least in part, by lifestyle modifications. Dietary salt consumption, a modifiable lifestyle factor, is associated not only with hypertension [15-19], but also cardiovascular diseases, independent of its effects on blood pressure $[20,21]$. Studies have reported a close relationship between excess salt intake and hypertension [15-19], and restriction of dietary salt reduces blood pressure in hypertensive patients [22, 23] and may prevent the development of hypertension in a normotensive population [24, 25]. Moreover, salt restriction slows the deterioration of kidney function in patients with CKD $[26,27]$ and may prevent the development of ESRD. This suggests that dietary salt plays a significant role in the age-dependent decline in kidney function in healthy individuals. However, the relationship between dietary salt and kidney function or the development of CKD in individuals with normal kidney function has not been studied intensively. Thus, the aim of the present study was to test the hypothesis that dietary salt intake predicts future impairment of kidney function in the general population.

\section{Materials and Methods}

\section{Study design}

The present cohort study investigating the effect of salt intake on kidney function was performed on participants attending their annual physical check-up. The study protocol conformed with the principles of the Declaration of Helsinki and the study was approved by the Ethics Committee of Enshu Hospital. All participants provided written informed consent prior to the start of the study and at each study visit.

\section{Study participants and procedures}

The present study enrolled 12126 consecutive subjects aged $\geq 20$ years with a normal estimated glomerular filtration rate (eGFR $\geq 60 \mathrm{~mL} / \mathrm{min}$ per $1.73 \mathrm{~m}^{2}$ ) who visited our hospital for their annual physical check-up between July 2008 and December 2015. As part of the annual health check-up, attendees are interviewed regarding their health status and undergo routine physical examination, chest X-ray, electrocardiography, and laboratory assessment of cardiovascular risk factors. Study participants were followed-up until December 2016 (median 1754 days), with the endpoint being the onset of kidney impairment (eGFR $<60 \mathrm{~mL} / \mathrm{min}$ per $1.73 \mathrm{~m}^{2}$ ). For each annual checkup, participants were instructed to collect overnight urine into a paper cup and to bring a sample of the urine in a plastic tube. Salt intake was assessed by estimating 24-h urinary sodium excretion, calculated using the Kamata formula [28]. This formula estimates 24-h sodium excretion using overnight urine and lean body mass in the Japanese population, and the correlation coefficient between measured and estimated sodium excretion is 0.78 [28]. Changes in kidney function were assessed using the function $Y=f(X)$, where $Y$ is eGFR and $X$ is the follow-up period (years). Linear regression analysis was performed for each participant using eGFR as a dependent variable ( $y$-axis) and follow-up period (years) as an independent variable ( $x$-axis); the slope of the regression 


\section{Kidney Blood Pressure Research}

Sugiura et al.: Salt and Chronic Kidney Disease

line was considered as the yearly change in eGFR $\left(\mathrm{mL} / \mathrm{min}\right.$ per $1.73 \mathrm{~m}^{2}$ per year). For participants who developed impaired kidney function during the study period, changes in eGFR were calculated using data obtained between enrollment and the onset of kidney function impairment. The effects of baseline salt intake on the onset of impaired kidney function (eGFR $<60 \mathrm{~mL} / \mathrm{min}$ per $1.73 \mathrm{~m}^{2}$ ) or on the yearly change in eGFR were investigated. Blood pressure was measured by trained technicians using a standard mercury sphygmomanometer in the morning after overnight fasting in seated participants. Three consecutive blood pressure measurements were taken at 2-min intervals, and the mean of the second and third measurements was recorded as the blood pressure. Hypertension was defined as systolic blood pressure $\geq 140 \mathrm{mmHg}$, diastolic blood pressure $\geq 90 \mathrm{mmHg}$, or the use of antihypertensive medications [29]. Diabetes mellitus was defined as fasting plasma glucose $\geq 126 \mathrm{mg} / \mathrm{dL}$ or the use of antidiabetic medications, and dyslipidemia was defined as low-density lipoprotein cholesterol $\geq 140 \mathrm{mg} / \mathrm{dL}$, high-density lipoprotein cholesterol $<40 \mathrm{mg}$ / $\mathrm{dL}$, triglyceride $\geq 150 \mathrm{mg} / \mathrm{dL}$, or the use of antidyslipidemic medications [30]. The eGFR was calculated using the modified Modification of Diet in Renal Disease study formula for the Japanese population [31]. Urinary protein was determined using a dipstick method (Arkray, Kyoto, Japan), which was interpreted by trained technicians and recorded as,, $- \pm 1+, 2+, 3+$. Proteinuria was defined as a result of $1+, 2+$, or $3+$. Participants reported alcohol consumption as ranging between 0 and 7 times/week, with frequent alcohol consumption defined as 6 or 7 times/week.

\section{Statistical analysis}

All analyses were performed using IBM SPSS statistics 24 (IBM SPSS, Chicago, IL, USA). Data are presented as the mean \pm SD or as the number and percentage of participants, except for the follow-up period. The significance of differences between two means with a normal distribution was determined using Student's unpaired $t$-test. Chi-squared tests were used for comparisons of categorical data. The Kaplan-Meier method was used to calculate the cumulative incidence rates of new kidney impairment. The significance of differences in cumulative incidence rates was evaluated by the log-rank test and adjusted by multivariate Cox proportional hazard regression models. Hazard ratios (HRs) and 95\% confidence intervals (CIs) were calculated. The relationship between salt intake as a continuous variable and the onset of kidney impairment was investigated using multivariate Cox proportional hazard regression models. The effect of baseline salt intake on changes in eGFR was assessed by multivariate linear regression analysis. In all cases, two-sided $P<0.05$ was considered significant.

\section{Results}

The baseline characteristics of participants are given in Table 1. Salt intake was greater in male than female participants $(12.1 \pm 3.1$ vs. $8.3 \pm 2.2 \mathrm{~g}$ /day; $P<0.001)$, but eGFR was lower in males than females $\left(80.3 \pm 12.6\right.$ vs. $81.5 \pm 13.4 \mathrm{~mL} / \mathrm{min}$ per $\left.1.73 \mathrm{~m}^{2} ; P<0.001\right)$. The actual follow-up period in the present study was 55073 person-years, with a median follow-up period per participant of 1754 days (range 113-2999 days). During the followup period, 1384 participants developed impaired kidney function $(11.4 \%$; 25.2 per 1000 person-years); the incidence of impaired kidney function was higher in male than female participants (12.1\% [26.2 per 1000 person-years] vs. 10.4\% [23.4 per 1000 person-years], respectively; $P<0.05$ ). Retrospective analysis revealed that salt intake was higher in subjects who subsequently developed impaired kidney function than in those who did not $11.2 \pm$ 3.4 vs. $10.6 \pm 3.4 \mathrm{~g}$ /day, respectively; $P<0.001$ ), although most other variables also differed significantly at baseline between these two groups (Table 2).

Univariate and multivariate Cox hazard regression analysis adjusted for possible risk factors, including eGFR at baseline, revealed that baseline salt intake (as a continuous variable) was an independent predictor of future kidney impairment (Table 3; all variables included in the multivariate model are listed in the table). In the next series of analyses, participants were divided into groups according to salt intake at baseline and the effect of salt intake on subsequent kidney function was investigated. First, participants were divided into two groups using the salt intake targets recommended by the Japanese Ministry of Health, Labour, 


\section{Kidney Blood Pressure Research}

Kidney Blood Press Res 2018;43:1245-1254

\begin{tabular}{l|l}
\hline DOI: $10.1159 / 000492406$ & (C) 2018 The Author(s). Published by S. Karger AG, Basel
\end{tabular}

Published online: 3 August, 2018

www.karger.com/kb

1248 and Welfare for a healthy population as the cut-off levels (men <8.0 g/day; women $<7.0$ $\mathrm{g} /$ day). The characteristics of these two groups are given in Table 4. Salt intake was approximately twofold higher in the highthan low-salt intake group. The incidence of kidney impairment in the low- and high-salt intake groups was 17.8 and 26.5 per 1000 person-years, respectively, and there was a significant difference in the cumulative incidence rate of kidney impairment as determined by the Kaplan-Meier method (Fig. 1). Multivariate Cox hazard regression analysis indicated an approximate $29 \%$ increase in the risk of developing impaired kidney function in participants with a higher salt intake (Table 5, Model A). Participants were then divided into gender-specific quartiles according to baseline salt intake, and the incidence of kidney impairment in each of the groups was analyzed. Salt intake in the first $(\mathrm{Q} 1)$, second $(\mathrm{Q} 2)$, third $(\mathrm{Q} 3)$, and fourth quartiles $(\mathrm{Q} 4)$ was $5.7 \pm 0.8$, $7.4 \pm 0.4,8.8 \pm 0.4$ and $11.2 \pm 1.5 \mathrm{~g} /$ day in female and $8.4 \pm 1.2,10.9 \pm 0.6,12.8 \pm 0.6$ and $16.3 \pm 2.1 \mathrm{~g} /$ day in male participants, respectively, and the incidence of impaired kidney function increased across the quartiles (19.0, 22.2, 29.3, and 30.4 per 1000 person-years in Q1, Q2, Q3, and Q4, respectively; $P<0.001$, log-rank test). Multivariate Cox hazard regression analysis revealed that the HR increased with increasing salt intake (Table 5, Model B). Both univariate and multivariate liner regression analyses indicated a significant correlation between salt intake at baseline and yearly changes in eGFR during the follow-up period (Table 6).

Similar results to those reported in Tables 3, 5, and 6 were obtained in subanalyses of participants without proteinuria $(\mathrm{n}=11903)$, hypertension ( $\mathrm{n}$ = 9113), diabetes mellitus $(n=11265)$, or those not using any medications for hypertension, diabetes mellitus, and dyslipidemia ( $\mathrm{n}=9562$ ) at baseline (data not shown). Furthermore, in subanalyses where CKD was defined as eGFR $<60 \mathrm{~mL} / \mathrm{min}$ per $1.73 \mathrm{~m}^{2}$ and/or the presence of proteinuria, baseline salt intake was found to be an independent
Table 1. Baseline characteristics of the study subjects $(n=12126)$. Data are given as the mean $\pm \mathrm{SD}$ or as $\mathrm{n}(\%)$. ${ }^{\mathrm{A}}$ Frequent alcohol consumption was defined as the consumption of alcohol six or seven times per week. SBP, systolic blood pressure; DBP, diastolic blood pressure; FPG, fasting plasma glucose; eGFR, estimated glomerular filtration rate; LDL-C, low-density lipoprotein cholesterol; HDL-C, high-density lipoprotein cholesterol

\begin{tabular}{lc}
\hline Parameter & Value \\
\hline Age (years) & $52.1 \pm 11.9$ \\
No. males & $7433(61.3)$ \\
Body mass index $\left(\mathrm{kg} / \mathrm{m}^{2}\right)$ & $22.7 \pm 3.3$ \\
SBP (mmHg) & $123.3 \pm 15.8$ \\
DBP (mmHg) & $75.9 \pm 9.9$ \\
Heart rate (b.p.m.) & $63.4 \pm 9.4$ \\
Serum creatinine (mg/dL) & $0.74 \pm 0.14$ \\
eGFR (mL/min per $\left.1.73 \mathrm{~m}^{2}\right)$ & $80.8 \pm 12.9$ \\
Uric acid (mg/dL) & $5.3 \pm 1.4$ \\
FPG (mg/dL) & $96.8 \pm 19.0$ \\
LDL-C (mg/dL) & $120.4 \pm 28.7$ \\
HDL-C (mg/dL) & $60.0 \pm 14.3$ \\
Triglyceride (mg/dL) & $108.9 \pm 78.2$ \\
Hemoglobin (g/dL) & $14.0 \pm 1.4$ \\
Salt intake (g/day) & $10.6 \pm 3.4$ \\
Proteinuria & $223(1.8)$ \\
Frequent alcohol consumption & \\
Current smoking status & $3730(30.8)$ \\
Yearly decline in eGFR (mL/min per $1.73 \mathrm{~m}^{2}$ per year) & $2984(24.6)$ \\
Hypertension & $1.18 \pm 4.40$ \\
Diabetes mellitus & $3013(24.8)$ \\
Dyslipidemia & $861(7.1)$ \\
\end{tabular}

Table 2. Retrospective analysis of subject characteristics at baseline according to the development of impaired kidney function. Data are given as the mean \pm SD or as $\mathrm{n}(\%) .{ }^{*} \mathrm{P}<0.05,{ }^{* *} \mathrm{P}<0.001$ compared with subjects who did not develop impaired kidney function (unpaired Student's t-test or Chi-squared test [gender, proteinuria, frequent alcohol consumption, current smoking status, hypertension, diabetes mellitus, dyslipidemia]). SBP, systolic blood pressure; DBP, diastolic blood pressure; FPG, fasting plasma glucose; eGFR, estimated glomerular filtration rate; LDL-C, low-density lipoprotein cholesterol; HDL-C, high-density lipoprotein cholesterol

\begin{tabular}{lcc}
\hline \multirow{2}{*}{ Parameter } & Developed impaired kidney function \\
& No $(\mathrm{n}=10742)$ & Yes $(\mathrm{n}=1384)$ \\
\hline Age (years) & $51.4 \pm 11.8$ & $58.0 \pm 10.9^{* *}$ \\
No. males & $6537(60.9)$ & $896(64.7)^{\dagger}$ \\
Body mass index $\left(\mathrm{kg} / \mathrm{m}^{2}\right)$ & $22.6 \pm 3.3$ & $23.1 \pm 3.1^{* *}$ \\
SBP (mmHg) & $122.8 \pm 15.6$ & $127.3 \pm 16.7$ \\
DBP (mmHg) & $75.7 \pm 9.8$ & $77.7 \pm 10.2^{* *}$ \\
Heart rate (b.p.m.) & $63.4 \pm 9.3$ & $63.3 \pm 9.9$ \\
Serum creatinine $(\mathrm{mg} / \mathrm{dL})$ & $0.72 \pm 0.13$ & $0.84 \pm 0.13^{* *}$ \\
eGFR (mL/min per $\left.1.73 \mathrm{~m}^{2}\right)$ & $82.4 \pm 12.6$ & $67.7 \pm 6.0^{* *}$ \\
Uric acid (mg/dL) & $5.3 \pm 1.4$ & $5.7 \pm 1.4^{* *}$ \\
FPG (mg/dL) & $96.6 \pm 18.5$ & $99.1 \pm 21.9^{* *}$ \\
LDL-C (mg/dL) & $120.2 \pm 28.9$ & $122.3 \pm 27.4{ }^{\dagger}$ \\
HDL-C (mg/dL) & $60.2 \pm 14.4$ & $58.1 \pm 13.7^{* *}$ \\
Triglyceride (mg/dL) & $107.9 \pm 78.8$ & $117.0 \pm 73.3^{* *}$ \\
Hemoglobin (g/dL) & $14.0 \pm 1.4$ & $13.9 \pm 1.4 \dagger$ \\
Salt intake (g/day) & $10.6 \pm 3.4$ & $11.2 \pm 3.4^{* *}$ \\
Proteinuria & $196(1.8)$ & $27(2.0)$ \\
Frequent alcohol consumption & $3313(30.8)$ & $417(30.1)$ \\
Current smoking status & $2733(25.4)$ & $251(18.1)^{* *}$ \\
Yearly decline in eGFR (mL/min per $1.73 \mathrm{~m}^{2}$ per year) & $0.71 \pm 4.11$ & $4.89 \pm 4.82^{* *}$ \\
Hypertension & $2470(23.0)$ & $543(39.2)^{* *}$ \\
Diabetes mellitus & $721(6.7)$ & $140(10.1)^{* *}$ \\
Dyslipidemia & $4351(40.5)$ & $668(48.3)^{* *}$ \\
\hline
\end{tabular}




\section{Kidney Blood Pressure Research}

predictor of the development of CKD or the decline of eGFR in individuals without CKD at baseline ( $\mathrm{n}=11$ 903; data not shown).

Table 3. Cox proportional hazard regression analyses for the development of impaired kidney function. All variables included in the multivariate analysis are listed in the table. $\mathrm{HR}$, hazard ratio; $\mathrm{CI}$, confidence interval; SBP, systolic blood pressure; DBP, diastolic blood pressure; FPG, fasting plasma glucose; eGFR, estimated glomerular filtration rate; LDL-C, low-density lipoprotein cholesterol; HDL-C, high-density lipoprotein cholesterol

\begin{tabular}{|c|c|c|c|c|}
\hline \multirow{2}{*}{ Variable at baseline } & \multicolumn{2}{|l|}{ Univariate } & \multicolumn{2}{|c|}{ Multivariate } \\
\hline & HR $(95 \% \mathrm{CI})$ & $\mathrm{P}$ value & HR $(95 \% \mathrm{CI})$ & $P$ value \\
\hline Age (years) & 1.049 (1.044-1.055) & $<0.001$ & $1.010(1.004-1.016)$ & 0.001 \\
\hline Male gender & $1.113(0.997-1.243)$ & 0.055 & $0.989(0.822-1.191)$ & 0.911 \\
\hline Body mass index $\left(\mathrm{kg} / \mathrm{m}^{2}\right)$ & $1.036(1.020-1.052)$ & $<0.001$ & $0.993(0.973-1.013)$ & 0.474 \\
\hline SBP (mmHg) & $1.015(1.012-1.018)$ & $<0.001$ & $1.005(1.001-1.008)$ & 0.015 \\
\hline DBP (mmHg) & $1.015(1.010-1.021)$ & $<0.001$ & - & - \\
\hline Heart rate (b.p.m.) & $1.003(0.998-1.009)$ & 0.233 & $1.005(1.000-1.011)$ & 0.068 \\
\hline Serum creatinine (mg/dL) & $1.846(1.769-1.927)$ & $<0.001$ & - & - \\
\hline eGFR $\left(\mathrm{mL} / \mathrm{min}\right.$ per $\left.1.73 \mathrm{~m}^{2}\right)$ & $0.827(0.820-0.835)$ & $<0.001$ & $0.834(0.826-0.842)$ & $<0.001$ \\
\hline Uric acid $(\mathrm{mg} / \mathrm{dL})$ & $1.217(1.172-1.264)$ & $<0.001$ & $1.082(1.030-1.136)$ & 0.002 \\
\hline FPG $(\mathrm{mg} / \mathrm{dL})$ & 1.005 (1.003-1.008) & $<0.001$ & $1.005(1.002-1.008)$ & 0.002 \\
\hline LDL-C (mg/dL) & $1.003(1.001-1.005)$ & 0.003 & $0.999(0.997-1.001)$ & 0.509 \\
\hline HDL-C (mg/dL) & $0.991(0.987-0.995)$ & $<0.001$ & $0.997(0.993-1.002)$ & 0.245 \\
\hline Triglyceride (mg/dL) & $1.001(1.000-1.001)$ & 0.001 & - & - \\
\hline Hemoglobin $(\mathrm{g} / \mathrm{dL})$ & $0.972(0.938-1.008)$ & 0.130 & $0.908(0.863-0.955)$ & $<0.001$ \\
\hline Salt intake (g/day) & $1.047(1.032-1.062)$ & $<0.001$ & $1.045(1.025-1.065)$ & $<0.001$ \\
\hline Proteinuria & $1.178(0.805-1.724)$ & 0.412 & $1.602(1.086-2.364)$ & 0.017 \\
\hline Frequent alcohol consumption & $0.882(0.786-0.990)$ & 0.031 & $0.846(0.741-0.967)$ & 0.014 \\
\hline Current smoking status & $0.658(0.574-0.754)$ & $<0.001$ & $1.020(0.878-1.186)$ & 0.792 \\
\hline Hypertension & $1.996(1.792-2.232)$ & $<0.001$ & - & - \\
\hline Diabetes mellitus & $1.461(1.227-1.740)$ & $<0.001$ & - & - \\
\hline Dyslipidemia & $1.355(1.219-1.505)$ & $<0.001$ & - & - \\
\hline
\end{tabular}

Table 4. Baseline characteristics of study subjects divided into groups according to salt intake ${ }^{\mathrm{A}}$ Data are given as the mean $\pm \mathrm{SD}$ or as $\mathrm{n}(\%) .{ }^{*} \mathrm{P}<0.05,{ }^{* *} \mathrm{P}<0.001$ compared with the low-salt intake group (unpaired Student's t-test or Chi-squared test [gender, proteinuria, frequent alcohol consumption, current smoking status, hypertension, diabetes mellitus, dyslipidemia]). ${ }^{\text {AS }}$ ubjects were divided into two groups using the salt intake targets recommended by the Japanese Ministry of Health, Labour, and Welfare (men $<8.0 \mathrm{~g} /$ day; women $<7.0 \mathrm{~g}$ /day). SBP, systolic blood pressure; DBP, diastolic blood pressure; FPG, fasting plasma glucose; eGFR, estimated glomerular filtration rate; LDL-C, low-density lipoprotein cholesterol; HDL-C, high-density lipoprotein cholesterol

\begin{tabular}{|c|c|c|}
\hline \multirow{2}{*}{ Parameter } & \multicolumn{2}{|c|}{ Salt intake } \\
\hline & Low $(n=1914)$ & $\operatorname{High}(\mathrm{n}=10212)$ \\
\hline Age (years) & $48.1 \pm 11.9$ & $52.9 \pm 11.8^{* *}$ \\
\hline Male gender & $536(28.0)$ & $6897(67.5)^{* *}$ \\
\hline Body mass index $\left(\mathrm{kg} / \mathrm{m}^{2}\right)$ & $21.2 \pm 3.0$ & $22.9 \pm 3.3^{* *}$ \\
\hline SBP (mmHg) & $117.0 \pm 14.9$ & $124.5 \pm 15.7^{* *}$ \\
\hline $\mathrm{DBP}(\mathrm{mmHg})$ & $72.0 \pm 9.4$ & $76.6 \pm 9.8^{* *}$ \\
\hline Heart rate (b.p.m.) & $64.2 \pm 9.1$ & $63.3 \pm 9.4^{* *}$ \\
\hline Serum creatinine $(\mathrm{mg} / \mathrm{dL})$ & $0.68 \pm 0.13$ & $0.75 \pm 0.14^{* *}$ \\
\hline eGFR $\left(\mathrm{mL} / \mathrm{min}\right.$ per $\left.1.73 \mathrm{~m}^{2}\right)$ & $81.8 \pm 12.8$ & $80.6 \pm 12.9^{* *}$ \\
\hline Uric acid (mg/dL) & $4.8 \pm 1.3$ & $5.4 \pm 1.4^{* *}$ \\
\hline $\mathrm{FPG}(\mathrm{mg} / \mathrm{dL})$ & $91.9 \pm 15.3$ & $97.8 \pm 19.4^{* *}$ \\
\hline LDL-C (mg/dL) & $115.4 \pm 28.0$ & $121.4 \pm 28.8^{* *}$ \\
\hline HDL-C (mg/dL) & $64.0 \pm 14.2$ & $59.2 \pm 14.2^{* *}$ \\
\hline Triglyceride $(\mathrm{mg} / \mathrm{dL})$ & $88.2 \pm 55.1$ & $112.8 \pm 81.2^{* *}$ \\
\hline Hemoglobin $(\mathrm{g} / \mathrm{dL})$ & $13.4 \pm 1.5$ & $14.1 \pm 1.4^{* *}$ \\
\hline Salt intake (g/day) & $6.2 \pm 1.0$ & $11.5 \pm 3.0^{* *}$ \\
\hline Proteinuria & $71(3.7)$ & $152(1.5)^{* *}$ \\
\hline Frequent alcohol consumption & $342(17.9)$ & $3388(33.2)^{* *}$ \\
\hline Current smoking status & $404(21.1)$ & $2580(25.3)^{* *}$ \\
\hline Yearly decline in eGFR (mL/min per $1.73 \mathrm{~m}^{2}$ per year) & $0.89 \pm 4.41$ & $1.24 \pm 4.40^{*}$ \\
\hline Hypertension & $277(14.5)$ & $2736(26.8)^{* *}$ \\
\hline Diabetes mellitus & $62(3.2)$ & $799(7.8)^{* *}$ \\
\hline Dyslipidemia & $590(30.8)$ & $4430(43.4)^{* *}$ \\
\hline
\end{tabular}




\section{Kidney Blood Pressure Research}

Fig. 1. Incidence rates of kidney impairment in participants divided into groups with high (-; bold straight line) and low (----; dotted line) salt intake based on salt intake targets for a healthy population recommended by the Japanese Ministry of Health, Labour, and Welfare (men $<8.0 \mathrm{~g} /$ day; women $<7.0 \mathrm{~g} /$ day). $\mathrm{P}<0.001$ (log-rank test).

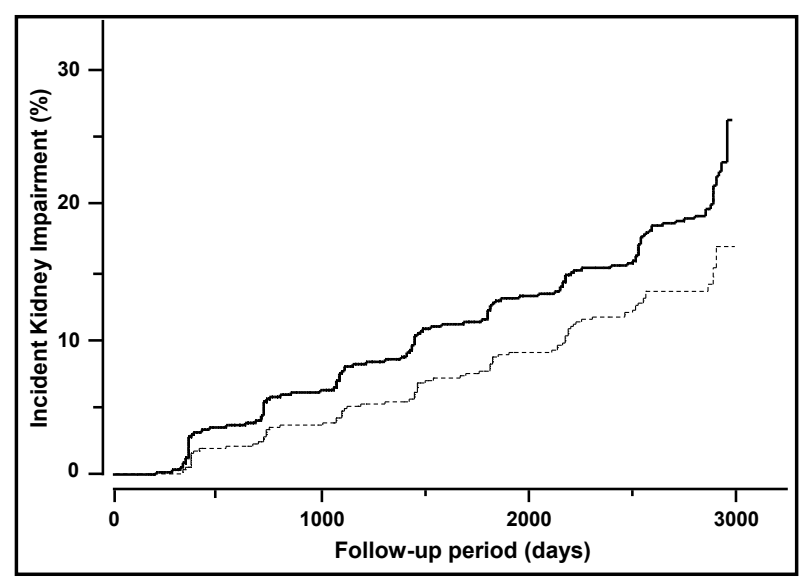

Table 5. Multivariate Cox proportional hazard regression analyses for the development of impaired kidney function. Model A, subjects were divided into high- and low-salt intake groups using the salt intake targets recommended by the Japanese Ministry of Health, Labour, and Welfare (men $<8.0 \mathrm{~g} /$ day; women $<7.0 \mathrm{~g}$ / day) as a cut-off level.Model B, subjects were divided into gender-specific quartiles according to salt intake. All variables included in the multivariate analysis are listed in the table. SBP, systolic blood pressure; DBP, diastolic blood pressure; FPG, fasting plasma glucose; eGFR, estimated glomerular filtration rate; LDL-C, low-density lipoprotein cholesterol; HDL-C, high-density lipoprotein cholesterol

\begin{tabular}{|c|c|c|c|c|}
\hline \multirow{2}{*}{ Variable at baseline } & \multicolumn{2}{|l|}{ Model A } & \multicolumn{2}{|l|}{ Model B } \\
\hline & $\operatorname{HR}(95 \% \mathrm{CI})$ & $P$ value & HR $(95 \% \mathrm{CI})$ & $P$ value \\
\hline Age (years) & $1.011(1.006-1.017)$ & $<0.001$ & $1.010(1.004-1.016)$ & 0.001 \\
\hline Male gender & $1.132(0.955-1.341)$ & 0.153 & $1.076(0.995-1.390)$ & 0.058 \\
\hline Body mass index $\left(\mathrm{kg} / \mathrm{m}^{2}\right)$ & $1.000(0.980-1.020)$ & 0.991 & $0.994(0.975-1.015)$ & 0.586 \\
\hline SBP (mmHg) & $1.005(1.001-1.008)$ & 0.009 & $1.005(1.001-1.008)$ & 0.013 \\
\hline Heart rate (b.p.m.) & $1.005(1.000-1.011)$ & 0.071 & $1.005(1.000-1.011)$ & 0.064 \\
\hline eGFR $\left(\mathrm{mL} / \mathrm{min}\right.$ per $\left.1.73 \mathrm{~m}^{2}\right)$ & $0.835(0.827-0.843)$ & $<0.001$ & $0.834(0.826-0.842)$ & $<0.001$ \\
\hline Uric acid $(\mathrm{mg} / \mathrm{dL})$ & $1.075(1.024-1.129)$ & 0.003 & $1.079(1.028-1.133)$ & 0.002 \\
\hline $\mathrm{FPG}(\mathrm{mg} / \mathrm{dL})$ & $1.005(1.002-1.008)$ & 0.001 & $1.005(1.002-1.008)$ & 0.002 \\
\hline LDL-C (mg/dL) & $0.999(0.997-1.001)$ & 0.391 & $0.999(0.997-1.001)$ & 0.463 \\
\hline $\mathrm{HDL}-\mathrm{C}(\mathrm{mg} / \mathrm{dL})$ & $0.997(0.993-1.002)$ & 0.246 & $0.997(0.993-1.002)$ & 0.233 \\
\hline Hemoglobin $(\mathrm{g} / \mathrm{dL})$ & $0.904(0.859-0.951)$ & $<0.001$ & $0.907(0.863-0.954)$ & $<0.001$ \\
\hline Proteinuria & $1.544(1.046-2.278)$ & 0.029 & $1.615(1.094-2.383)$ & 0.016 \\
\hline Frequent alcohol consumption & $0.851(0.745-0.972)$ & 0.017 & $0.853(0.747-0.974)$ & 0.019 \\
\hline Current smoking status & $0.998(0.859-1.159)$ & 0.981 & $1.020(0.878-1.185)$ & 0.800 \\
\hline High (vs. low) salt intake & $1.292(1.085-1.540)$ & 0.004 & - & - \\
\hline \multicolumn{5}{|l|}{ Salt intake quartiles } \\
\hline Q1 & - & - & 1 (reference) & - \\
\hline Q2 & - & - & $1.192(1.010-1.406)$ & 0.037 \\
\hline Q3 & - & - & 1.339 (1.142-1.569) & $<0.001$ \\
\hline Q4 & - & - & $1.458(1.240-1.714)$ & $<0.001$ \\
\hline
\end{tabular}

Table 6. Univariate and multivariate regression analyses demonstrating the relationship between baseline variables and yearly changes in estimated glomerular filtration rate. All variables included in multivariate analysis are listed in the table. SBP, systolic blood pressure; DBP, diastolic blood pressure; FPG, fasting plasma glucose; eGFR, estimated glomerular filtration rate; LDL-C, lowdensity lipoprotein cholesterol; HDL-C, high-density lipoprotein cholesterol

\begin{tabular}{lcccc}
\hline \multirow{2}{*}{ Variable at baseline } & \multicolumn{3}{c}{ Univariate } & \multicolumn{2}{c}{ Multivariate } \\
& $\beta$ & $\mathrm{P}$ value & $\beta$ & $\mathrm{P}$ value \\
\hline Age (years) & 0.012 & 0.188 & -0.012 & 0.266 \\
Male gender & 0.008 & 0.355 & 0.011 & 0.477 \\
Body mass index $\left(\mathrm{kg} / \mathrm{m}^{2}\right)$ & -0.035 & $<0.001$ & -0.017 & 0.123 \\
SBP $(\mathrm{mmHg})$ & -0.035 & $<0.001$ & -0.025 & 0.016 \\
DBP $(\mathrm{mmHg})$ & 0.012 & 0.187 & - & - \\
Heart rate (b.p.m.) & -0.038 & $<0.001$ & -0.018 & 0.057 \\
eGFR (mL/min per $\left.1.73 \mathrm{~m}^{2}\right)$ & -0.144 & $<0.001$ & -0.154 & $<0.001$ \\
Uric acid (mg/dL) & 0.005 & 0.620 & -0.043 & $<0.001$ \\
FPG (mg/dL) & -0.042 & $<0.001$ & -0.028 & 0.005 \\
LDL-C (mg/dL) & 0.029 & 0.001 & 0.034 & $<0.001$ \\
HDL-C (mg/dL) & 0.023 & 0.011 & 0.021 & 0.036 \\
Triglyceride (mg/dL) & 0.001 & 0.914 & - & - \\
Hemoglobin (g/dL) & 0.037 & $<0.001$ & 0.064 & $<0.001$ \\
Salt intake (g/day) & -0.051 & $<0.001$ & -0.060 & $<0.001$ \\
Proteinuria & -0.002 & 0.804 & 0.001 & 0.982 \\
Frequent alcohol consumption & 0.026 & 0.005 & 0.038 & $<0.001$ \\
Current smoking status & 0.016 & 0.078 & 0.013 & 0.179 \\
\hline
\end{tabular}




\section{Kidney Blood Pressure Research}

Kidney Blood Press Res 2018;43:1245-1254

\begin{tabular}{l|l}
\hline DOI: 10.1159/000492406 & (C) 2018 The Author(s). Published by S. Karger AG, Base
\end{tabular}

Published online: 3 August, 2018

www.karger.com/kb

Sugiura et al.: Salt and Chronic Kidney Disease

\section{Discussion}

The present observational study demonstrated that, in the general population with normal kidney function, dietary salt is a significant determinant of future kidney function independent of its effects on blood pressure. An increase in salt intake may accelerate the age-dependent decline in kidney function in the general population.

The findings of the present study propose an important concept, namely that dietary salt intake is closely associated with future kidney function in the general population. Individuals who developed kidney impairment during the observation period had a greater salt intake at baseline than those who did not. This finding from the present retrospective analysis was not very impressive, because the difference in the salt intake at baseline was relatively small and there were differences in most other factors investigated (i.e. age, blood pressure, and eGFR) between those who did and did not develop impaired kidney function. However, Cox hazard regression analysis clearly indicated that baseline salt intake was an independent predictor of the development of kidney impairment, defined as eGFR $<60 \mathrm{~mL} /$ min per $1.73 \mathrm{~m}^{2}$, regardless of the presence or absence of proteinuria. Multivariate analysis indicated that the risk of kidney impairment increased approximately $4.5 \%$ with each $1-\mathrm{g}$ increase in salt intake. In addition to salt intake, systolic blood pressure, plasma glucose, uric acid, and proteinuria were also significantly correlated with the incidence of impaired kidney function, confirming previous reports [32-34] and thus confirming the reliability of the results of the present study. The concept that salt intake is closely associated with the development of impaired kidney function was further reinforced by the findings of Kaplan-Meier analysis, which indicated that the risk of developing kidney impairment was increased in the group with the higher than lower salt intake, as well as across quartiles of salt intake. Cox proportional hazard regression analysis revealed that the risk of developing kidney impairment was increased approximately 29\% in the group with the higher salt intake (mean salt intake $11.5 \mathrm{~g}$ ) than in the group with lower salt intake (mean salt intake $6.2 \mathrm{~g}$ ), confirming the results of the multivariate analysis where salt intake was used as a continuous variable (Table 3 ) and showing an approximate $4.5 \%$ increase in the risk of developing kidney impairment with each 1-g increase in salt intake. The finding that baseline salt intake was closely and independently correlated with the yearly decline in eGFR also supports our hypothesis that dietary salt intake predicts future impairment of kidney function in the general population. The effects of lifestyle-related diseases or medications prescribed to the subjects in the present study on the results are likely minimal, because similar results were obtained in subanalyses in subjects without proteinuria, hypertension, diabetes mellitus, or not taking any medications for hypertension, diabetes mellitus, and dyslipidemia at baseline. The findings of the present study indicate that the lower the dietary salt intake at baseline, the better the kidney function going into the future. Although the salt intake targets recommended by the Japanese Ministry of Health, Labour, and Welfare for the healthy population (men $<8.0 \mathrm{~g} /$ day; women $<7.0 \mathrm{~g} /$ day) are acceptable for the present, these targets should not be considered final, taking into consideration that the average salt intake in the present study was $10.6 \mathrm{~g} /$ day. Of note, the subanalyses in the present study revealed that dietary salt is a significant predictor of incident CKD (defined as eGFR $<60$ $\mathrm{mL} / \mathrm{min}$ per $1.73 \mathrm{~m}^{2}$ and/or the presence of proteinuria) independent of its effects on blood pressure in the general population with normal kidney function, although urinary protein was not quantified in the present study and chronic proteinuria was not confirmed.

The mechanism underlying the close relationship between salt intake and the development of CKD cannot be clarified based on the findings of the present observational study. However, there are several likely mechanisms. Most of the sodium taken into the body is excreted by the kidneys through the mechanism of pressure-natriuresis [35-37]. Thus, excess salt intake may result in a pressure and volume overload of the kidney (nephrons), and the resulting glomerular hypertension may accelerate the decline in GFR or induce proteinuria. Indeed, proteinuria often reflects an increase in glomerular filtration pressure 


\section{Kidney Blood Pressure Research}

Sugiura et al.: Salt and Chronic Kidney Disease

and is an independent risk factor for a reduction in GFR [38-40], which was confirmed by the present study. The results of the present study and these speculations are compatible with previous reports that increased salt intake promotes a further decline in kidney function and the development of ESRD in patients with CKD [26, 27]. Mechanisms that involve endothelial dysfunction [41], activation of the sympathetic nervous system and the renin-angiotensin system within the kidney [42-45], and the transcription of mineralocorticoid receptordependent genes [44] may also be involved in the relationship between salt intake and the development of CKD.

The interpretation of the results of the present study is limited by the following. First, the study subjects were participants undergoing an annual physical check-up, so urine and blood samples, as well as blood pressure measurements, were only obtained once a year. Second, salt intake was estimated using a spot urine specimen. For estimating salt excretion, 24-h urinary measurement is the gold standard, but this approach is not practical for largescale studies. Because the difference between estimated and measured sodium excretion was reported to increase with increasing sodium excretion [28], the formula used in the present study tended to underestimate sodium excretion in participants with relatively high sodium excretion. Third, there is large within-individual and day-to-day variability in dietary salt intake. Fourth, the observational nature of the present study does not enable us to clarify the mechanisms underlying the results. These points should be considered when interpreting the data presented herein.

\section{Conclusion}

Dietary salt intake, independent of its effects on blood pressure, is significantly associated with the development of CKD and the yearly decline in eGFR in the general population, even in normotensive subjects. These results suggest that salt restriction is beneficial for the prevention of impaired kidney function and the development of CKD, and thereby cardiovascular diseases, in the general population.

\section{Disclosure Statement}

The authors declare they have no conflicts of interest regarding the publication of this paper.

\section{References}

$>1$ Centers for Disease Control and Prevention (CDC): Prevalence of chronic kidney disease and associated risk factors - United States, 1999-2004. MMWR Morb Mortal Wkly Rep 2007;56:161-165.

-2 Imai E, Horio M, Watanabe T, Iseki K, Yamagata K, Hara S, Ura N, Kiyohara Y, Moriyama T, Ando Y, Fujimoto S, Konta T, Yokoyama H, Makino H, Hishida A, Matsuo S.Prevalence of chronic kidney disease in the Japanese general population. Clin Exp Nephrol 2009;13:621-630.

-3 Lysaght MJ. Maintenance dialysis population dynamics: current trends and long-term implications. J Am Soc Nephrol 2002;13:S37-S40.

4 Go AS, Chertow GM, Fan D, McCulloch CE, Hsu CY: Chronic kidney disease and the risks of death, cardiovascular events, and hospitalization. N Engl J Med 2004;351:1296-1305.

5 Hsieh MC, Hsiao JY, Tien KJ, Chang SJ, Hsu SC, Liang HT, Chen HC, Lin SR, Tu ST: Chronic kidney disease as a risk factor for coronary artery disease in Chinese with type 2 diabetes. Am J Nephrol 2008;28:317-323.

6 Ryan TP, Fisher SG, Elder JL, Winters PC, Beckett W, Tacci J, Sloand JA: Increased cardiovascular risk associated with reduced kidney function. Am J Nephrol 2009;29:620-625. 


\section{Kidney \\ Blood Pressure Research}

Sugiura et al.: Salt and Chronic Kidney Disease

7 Nakayama M, Sato T, Sato H, Yamaguchi Y, Obara K, Kurihara I, Sato K, Hotta O, Seino J, Miyata M, Takeuchi K, Nakayama K, Matsushima M, Otaka T, Kinoshita Y, Taguma Y, Ito S: Different clinical outcomes for cardiovascular events and mortality in chronic kidney disease according to underlying renal disease: the Gonryo study. Clin Exp Nephrol 2010;14:333-339.

-8 Nagai K, Sairenchi T, Irie F, Watanabe H, Ota H, Yamagata K. Relationship between estimated glomerular filtration rate and cardiovascular mortality in a Japanese cohort with long-term follow-up. PLoS One 2016;11:e0156792.

\$ Keith DS, Nichols GA, Gullion CM, Brown JB, Smith DH: Longitudinal follow-up and outcomes among a population with chronic kidney disease in a large managed care organization. Arch Intern Med 2004;164:659663.

10 Fox CS, Larson MG, Leip EP, Culleton B, Wilson PW, Levy D: Predictors of new-onset kidney disease in a community-based population. JAMA 2004;291:844-850.

11 Hippisley-Cox J, Coupland C: Predicting the risk of chronic kidney disease in men and women in England and Wales: Prospective derivation and external validation of the QKidney Scores. BMC Fam Pract 2010;11:49.

12 O'Seaghdha CM, Lyass A, Massaro JM, Meigs JB, Coresh J, D’Agostino RB, Sr, Astor BC, Fox CS: A risk score for chronic kidney disease in the general population. Am J Med 2012;125:270-277.

13 Nitta K, Okada K, Yanai M, Takahashi S: Aging and chronic kidney disease. Kidney Blood Press Res 2013;38:109-120.

14 Levey AS, Bosch JP, Lewis JB, Greene T, Rogers N, Roth D: A more accurate method to estimate glomerular filtration rate from serum creatinine: a new prediction equation. Modification of Diet in Renal Disease Study Group. Ann Intern Med 1999;130:461-470.

15 Meneely GR, Dahl LK: Electrolytes in hypertension: the effects of sodium chloride. The evidence from animal and human studies. Med Clin North Am 1961;45:271-283.

-16 Intersalt Cooperative Research Group: Intersalt: an international study of electrolyte excretion and blood pressure. Results for 24 hour urinary sodium and potassium excretion. BMJ 1988;297:319-328.

17 Mente A, O’Donnell MJ, Rangarajan S, McQueen MJ, Poirier P, Wielgosz A, Morrison H, Li W, Wang X, Di C, Mony P, Devanath A, Rosengren A, Oguz A, Zatonska K, Yusufali AH, Lopez-Jaramillo P, Avezum A, Ismail N, Lanas F, et al.: Association of urinary sodium and potassium excretion with blood pressure. N Engl J Med 2014;371:601-611.

18 Takase H, Sugiura T, Kimura G, Ohte N, Dohi Y: Dietary sodium consumption predicts future blood pressure and incident hypertension in the Japanese normotensive general population. J Am Heart Assoc 2015;4:e001959.

19 Hashimoto T, Takase H, Okado T, Sugiura T, Yamashita S, Kimura G, Ohte N, Dohi Y: Significance of adjusting salt intake by body weight in the evaluation of dietary salt and blood pressure. J Am Soc Hypertens 2016;10:647-655.

20 Alderman MH, Cohen H, Madhavan S: Dietary sodium intake and mortality: the national health and nutrition examination survey (NHANES I). Lancet 1998;351:781-785.

-21 Strazzullo P, D’Elia L, Kandala NB, Cappuccio FP: Salt intake, stroke, and cardiovascular disease: metaanalysis of prospective studies. BMJ 2009;339:b4567.

22 Whelton PK, Appel LJ, Espeland MA, Applegate WB, Ettinger WH Jr, Kostis JB, Kumanyika S, Lacy CR, Johnson KC, Folmar S, Cutler JA: Sodium reduction and weight loss in the treatment of hypertension in older persons: a randomized controlled trial of nonpharmacologic interventions in the elderly (TONE). TONE Collaborative Research Group. JAMA 1998;279:839-846.

-23 Sacks FM, Svetkey LP, Vollmer WM, Appel LJ, Bray GA, Harsha D, Obarzanek E, Conlin PR, Miller ER III, Simons-Morton DG, Karanja N, Lin PH, DASH-Sodium Collaborative Research Group: Effects on blood pressure of reduced dietary sodium and the Dietary Approaches to Stop Hypertension (DASH) diet. DASHSodium Collaborative Research Group. N Engl J Med 2001;344:3-10.

24 He FJ, MacGregor GA: Effect of modest salt reduction on blood pressure: a meta-analysis of randomized trials. Implications for public health. J Hum Hypertens 2002;16:761-770.

25 Dickinson HO, Mason JM, Nicolson DJ, Campbell F, Beyer FR, Cook JV, Williams B, Ford GA: Lifestyle interventions to reduce raised blood pressure: a systematic review of randomized controlled trials. J Hypertens 2006;24:215-233. 


\section{Kidney \\ Blood Pressure Research}

Sugiura et al.: Salt and Chronic Kidney Disease

-26 Cianciaruso B, Bellizzi V, Minutolo R, Tavera A, Capuano A, Conte G, De Nicola L: Salt intake and renal outcome in patients with progressive renal disease. Miner Electrolyte Metab 1998;24:296-301.

27 Buter H, Hemmelder MH, Navis G, de Jong PE, de Zeeuw D: The blunting of the antiproteinuric efficacy of ACE inhibition by high sodium intake can be restored by hydrochlorothiazide. Nephrol Dial Transplant 1998;13:1682-1685.

28 Kamata K, Tochikubo 0: Estimation of 24-h urinary sodium excretion using lean body mass and overnight urine collected by a pipe-sampling method. J Hypertens 2002;20:2191-2197.

-29 Shimamoto K, Ando K, Fujita T, Hasebe N, Higaki J, Horiuchi M, Imai Y, Imaizumi T, Ishimitsu T, Ito M, Ito S, Itoh H, Iwao H, Kai H, Kario K, Kashihara N, Kawano Y, Kim-Mitsuyama S, Kimura G, Kohara K, et al.: The Japanese Society of Hypertension Guidelines for the Management of Hypertension (JSH 2014). Hypertens Res 2014;37:253-390.

-30 Teramoto T, Sasaki J, Ueshima H, Egusa G, Kinoshita M, Shimamoto K, Daida H, Biro S, Hirobe K, Funahashi T, Yokote K, Yokode M, Japan Atherosclerosis Society (JAS) Committee for Epidemiology and Clinical Management of Atherosclerosis: Diagnostic criteria for dyslipidemia. Executive summary of Japan Atherosclerosis Society (JAS) guideline for diagnosis and prevention of atherosclerotic cardiovascular diseases for Japanese. J Atheroscler Thromb 2007;14:155-158.

-31 Matsuo S, Imai E, Horio M, Yasuda Y, Tomita K, Nitta K, Yamagata K, Tomino Y, Yokoyama H, Hishida A, Collaborators developing the Japanese equation for estimated GFR. Revised equations for estimated GFR from serum creatinine in Japan. Am J Kidney Dis 2009;53:982-992.

-32 Klag MJ, Whelton PK, Randall BL, Neaton JD, Brancati FL, Ford CE, Shulman NB, Stamler J: Blood pressure and end-stage renal disease in men. N Engl J Med 1996;334:13-18.

33 Yamagata K, Ishida K, Sairenchi T, Takahashi H, Ohba S, Shiigai T, Narita M, Koyama A: Risk factors for chronic kidney disease in a community-based population: a 10-year follow-up study. Kidney Int 2007;71:159-166.

-34 Sonoda H, Takase H, Dohi Y, Kimura G: Uric acid levels predict future development of chronic kidney disease. Am J Nephrol 2011;33:352-357.

35 Guyton AC, Coleman TG, Cowley AV, Scheel KW, Manning RD, Norman RA: Arterial pressure regulation. Overriding dominance of the kidneys in long-term regulation and in hypertension. Am J Med 1972;52:584594.

36 Guyton AC: Renal function curve--a key to understanding the pathogenesis of hypertension. Hypertension 1987;10:1-6.

-37 Guyton AC: Blood pressure control-special role of the kidneys and body fluids. Science 1991;252:1813-1816.

38 Jafar TH, Stark PC, Schmid CH, Landa M, Maschio G, Marcantoni C, de Jong PE, de Zeeuw D, Shahinfar S, Ruggenenti P, Remuzzi G, Levey AS, AIPRD Study Group: Proteinuria as a modifiable risk factor for the progression of non-diabetic renal disease. Kidney Int 2001;60:1131-1140.

39 Lea J, Greene T, Hebert L, Lipkowitz M, Massry S, Middleton J, Rostand SG, Miller E, Smith W, Bakris GL: The relationship between magnitude of proteinuria reduction and risk of end-stage renal disease: results of the African American study of kidney disease and hypertension. Arch Intern Med 2005;165:947-953.

-40 Halbesma N, Kuiken DS, Brantsma AH, Bakker SJ, Wetzels JF, De Zeeuw D, De Jong PE, Gansevoort RT: Macroalbuminuria is a better risk marker than low estimated GFR to identify individuals at risk for accelerated GFR loss in population screening. J Am Soc Nephrol 2006;17:2582-2590.

41 Boegehold MA: The effect of high salt intake on endothelial function: reduced vascular nitric oxide in the absence of hypertension. J Vasc Res 2013;50:458-467.

-42 Blaustein MP, Leenen FH, Chen L, Golovina VA, Hamlyn JM, Pallone TL, Van Huysse JW, Zhang J, Wier WG: How $\mathrm{NaCl}$ raises blood pressure: a new paradigm for the pathogenesis of salt-dependent hypertension. Am J Physiol Heart Circ Physiol 2012;302:H1031-1049.

43 Bolívar JJ: Essential hypertension: an approach to its etiology and neurogenic pathophysiology. Int J Hypertens 2013;2013:547809.

44 Fujita T: Mechanism of salt-sensitive hypertension: focus on adrenal and sympathetic nervous systems. J Am Soc Nephrol 2014;25:1148-1155.

45 Simmonds SS, Lay J, Stocker SD: Dietary salt intake exaggerates sympathetic reflexes and increases blood pressure variability in normotensive rats. Hypertension 2014;64:583-589. 